\title{
The Super Charm-Tau Factory in Novosibirsk
}

\section{A.Yu. Barnyakov*t}

Budker Institute of Nuclear Physics, 630090, pr. Lavrentieva 11, Novovsibirsk, Russia Novosibirsk State University, 630090, st. Pirogova 2, Novovsibirsk, Russia Novosibirsk State Technical University, 630092, pr. Karla Marksa 20, Novovsibirsk, Russia

E-mail: a.yu.barnyakoveinp.nsk.su

The Super Charm-Tau Factory is an electron-positron collider project in Novosibirsk with a peak luminosity of $10^{35} \mathrm{~cm}^{-1} \mathrm{~s}^{-1}$ operating in the center of mass energy range between 2 and $6 \mathrm{GeV}$. Longitudinal polarization of the electrons at the interaction region is foreseen in the project. The physics program of the experiment is devoted to the measurements of charm quark and tau lepton. It includes precision tests of the standard model in the electro-weak sector, QCD measurements in the non-perturbative region, and searches for the beyond standard model phenomena. Conceptual designs of the collider based on crab-waist technique and universal detector are developed and presented. The preliminary physics program of the project is considered. Progresses of the physics simulation and systems prototyping are given.

XXIX International Symposium on Lepton Photon Interactions at High Energies - LeptonPhoton2019 August 5-10, 2019

Toronto, Canada

\footnotetext{
${ }^{*}$ Speaker.

${ }^{\dagger}$ on behalf of Super Charm-Tau Factory collaboration
} 


\section{Introduction}

The Super Charm-Tau (SCT) Factory is an electron-positron collider project in Novosibirsk with a peak luminosity of $10^{35} \mathrm{~cm}^{-1} \mathrm{~s}^{-1}$ operating in the center of mass energy range between 2 and $6 \mathrm{GeV}$ [1]. The goal luminosity of the collider will exceed in 100 times the luminosity of BEPC-II (Beijing Electron-Positron Collider, China) [2], which is under operation today in the same energy range. The other feature of the project is a high level $(80-90 \%)$ of longitudinal polarization for the electron beam at the interaction point. The proposed collider machine with high luminosity and beam longitudinal polarization combined with high performance universal detector will allow us to provide a series of precise experiments and tests of Standard Model (SM).

According to European strategy for Particle Physics Update 2020 [3] precise experiments to search for new physics beyond the SM are very demanded in the near future. From this point of view the particle physics experiments at SCT Factory will compliment the physics at SuperKEKb collider (Japan) and Phase II (High-Luminosity) LHC (Switzerland). The physics results from all these experiments will help to formulate more precisely the future physics program of colliding beam experiments. Also the Super Charm-Tau Factory in Novosibirsk could be a very good facility to test and experimentally determine all advantages and critical issues of the new colliding beam method, called Crab-Waist (CW) technique.

\section{Some consideration of the preliminary physics program}

In Figure 1 the ratio $(R)$ of hadron production cross-section to muon production cross-section is shown for the energy range of the Novosibirsk SCT Factory. The operation energy range from $2 \mathrm{GeV}$ to $6 \mathrm{GeV}$ will cover the thresholds of $\tau$ - lepton production and almost all charmed states up to region of $\Omega_{c}$-baryon. The physics program of the experiment is devoted to the measurements of charm quark and tau lepton. It includes precision tests of the SM in the electro-weak sector (lepton flavor universality tests, measurement of the CKM matrix elements, measurements of the weak charged current structure in tau decays), QCD measurements in the non-perturbative region (hadronic cross sections, spectroscopy, dynamics of the hadronic decays of the charmed hadrons and tau lepton), and searches for the beyond SM phenomena (lepton flavour violating decays and other forbidden or highly suppressed in the SM processes). The possible approximate distribution

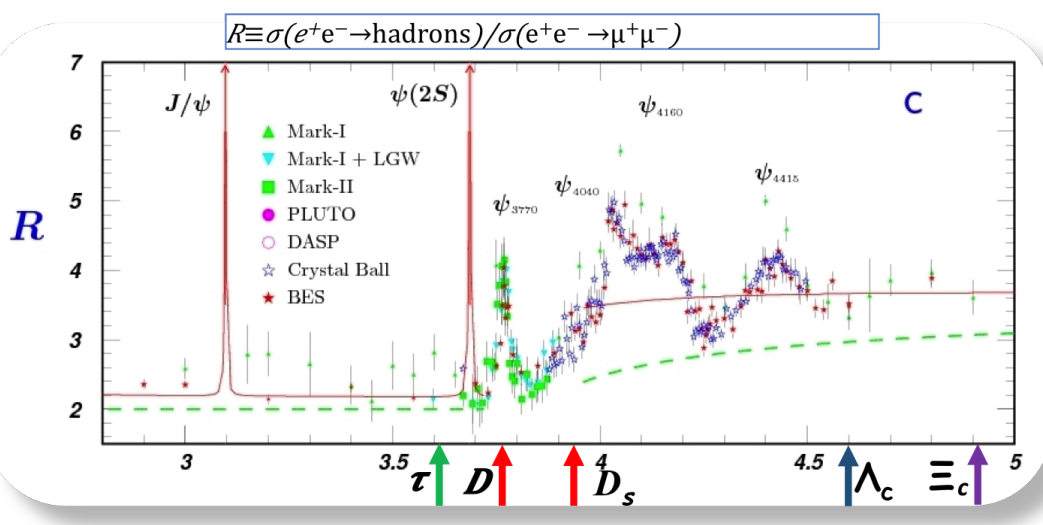

Figure 1: R ratio for SCT Factory operation energy range. 


\begin{tabular}{|c|c|c|c|}
\hline Energy, GeV & $\mathrm{L}, \mathrm{fb}^{-1}$ & & Comments \\
\hline 3.096 & 300 & $J / \psi$ & $\begin{array}{c}\text { rare decays } \\
\text { light meson spectroscopy }\end{array}$ \\
\hline 3.554 & 50 & $\begin{array}{l}e^{+} e^{-} \rightarrow \tau^{+} \tau^{-} \\
\quad(\text { threshold) }\end{array}$ & Precision measurements of $\tau$ decays \\
\hline 3.686 & 150 & $\psi(2 S)$ & $\begin{array}{c}J / \psi \text {-spectroscopy } \\
\text { light hadr. spectroscopy }\end{array}$ \\
\hline 3.770 & 300 & $\psi(3770)$ & (D-meson study) \\
\hline 4.170 & 100 & $\psi * 4160)$ & $\left(D_{s}\right.$-meson study) \\
\hline 4.650 & 100 & $\begin{array}{c}e^{+} e^{-} \rightarrow \Lambda_{c}^{+} \Lambda_{c}^{-} \\
(\operatorname{maximum})\end{array}$ & $\Lambda_{c}$ study \\
\hline
\end{tabular}

Table 1: Energies, at which most of data will be collected, and approximate distribution of the integrated luminosity collected during one experimental run $\left(10^{7} \mathrm{~s}, 1 \mathrm{ab}^{1}\right)$ over these energies.

of an integrated luminosity collected during one experimental run $\left(10^{10} \mathrm{~s}\right.$ and $\left.1 \mathrm{ab}^{-1}\right)$ is presented in Table 1.

During 10 years of operation of the SCT factory the integrated luminosity will be about $10 \mathrm{ab}^{-1}$, therefore $N_{\tau^{+} \tau^{-}}$could reach $2 \times 10^{10}$, while the expected total statistic at Super-B factory (Belle II experiment) $N_{\tau^{+} \tau^{-}} \sim 6.9 \times 10^{10}$. The total statistics of the $\mathrm{D}, \mathrm{D}^{*}$ and $\mathrm{D}_{s^{-}}$-mesons will also be significantly less than expected at the Super-B factory (Belle II experiment) and already collected at the LHCb experiment.

The project has several advantages which will help to perform a series of precise experiments:

- threshold production;

- well determined initial state;

- quantum correlated production of $\mathrm{D}^{0}$ meson pairs;

- double tag technique;

- low multiplicity (4-5);

- longitudinal beam polarization.

Some feasibility studies demonstrate that sensitivity of the SCT factory project to $\tau$-lepton physics will be better than at Super-B factory and at proton colliders. For instance, sensitivity to search for $\tau \rightarrow \mu \gamma$ process (LFV in $\tau$-decay) at SCT factory will be 10 times better than at Super-B due to better kinematics of the $\tau$ near the threshold of production and due to good $\mu / \pi$-separation in dedicated particle identification (PID) system [4, 5]. The longitudinal polarization of the electron beam better than $50 \%$ will help to perform more accurate measurements of the $\tau$-lepton Michel parameters than expected at Super-B factory [6]. The other feasibility studies for other physics cases now are under consideration.

\section{Collider concept}

The SCT factory is a symmetric double ring electron-positron collider. The high single-bunch luminosity is achieved by implementation of Crab-Waist collision scheme with a large Pivinsky parameter and submillimeter $\beta_{y}$ (vertical beta-function) in interaction region. Today only two collider projects with large Pivinsky parameter are under operation: DAФNE (LNF, Italy) [7] and SuperKEKb (KEK, Japan) [8]. In the first case this collision scheme helps to increase the luminosity approximately by 4 times, in the case of SuperKEKb project an increase of luminosity by 40 times is expected. The estimated effect from implementation of the new collision scheme for SCT factory is about a factor of 100 in comparison with the traditional one which is used at Chinese $\mathrm{C}-\tau$ factory BEPC-II (IHEP) [2]. 
The first collider concept was developed in 2011 and its main parameters are presented in [10]. In 2019 the concept of accelerating complex was updated. The sketch of the collider rings and major changes are presented in Figure 2.

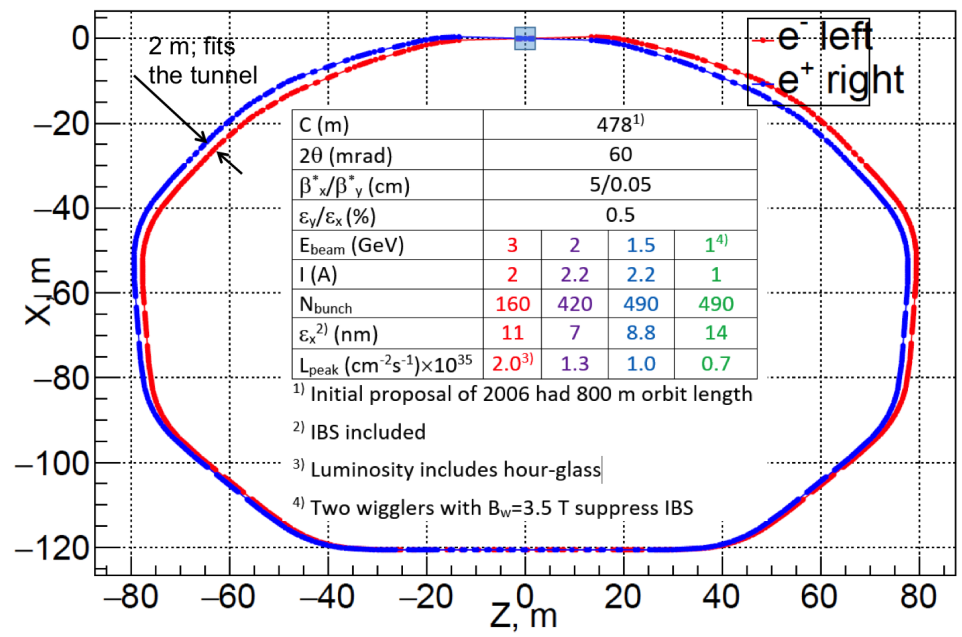

Figure 2: The scheme and main parameters of the collider rings for the Super Charm-Tau factory project.

The other feature of the SCT factory project in Novosibirsk is a longitudinal electron beam polarization in interaction region. It is shown in [9] that the level of 70-80\% electron beam polarization for beam energy range below $2.5 \mathrm{GeV}$ is achievable with help of three Sibirian Snake spin rotators. $50 \%$ polarization at $3 \mathrm{GeV}$ is expected in this scheme.

The modern accelerator complex concept for Novosibirsk SCT factory has no unsolved issues, prototyping of key components of the accelerator complex is being carried out at BINP.

\section{Detector}

The proposed broad physics program requires construction of a universal high performance magnetic detector with a field of $1-1.5 \mathrm{~T}$. The common requirements for the detector from physics program and collider parameters are following:

- good $\frac{\sigma_{P}}{P}$ for charged particles.

- good symmetry and hermeticity;

- soft track detection;

- good $\mu / \pi$-separation up to $1.5 \mathrm{GeV} / \mathrm{c}$ and $\pi / K$-separation up to $2.5 \mathrm{GeV} / \mathrm{c}$;

- good $\pi^{0} / \gamma$-separation and $\gamma$ detection with $\mathrm{E}_{\gamma}=10 \div 3000 \mathrm{MeV}$;

- fast enough readout electronics to work with trigger rate up to $300 \mathrm{kHz}$. The tracking system is divided in two parts: inner tracker to detect soft tracks (with momentum below $100 \mathrm{MeV} / \mathrm{c}$ ) and main tracker based on drift chamber (DC). For the inner tracker several different options are under consideration: Si-strip 4-layer detector, cylindrical GEM (Gas Electron Multiplayer) and TPC (Time-Projection-Chamber) [11]. The main limitation for use of different options close to the interaction region comes from physics background (BhaBha scattering and two gamma production of $\mathrm{e}^{+} \mathrm{e}^{-}$pairs). First results of the physics background simulation for the SCT factory project are presented in [12]. For DC there are two proposals. One of them is called "traditional" which is based 


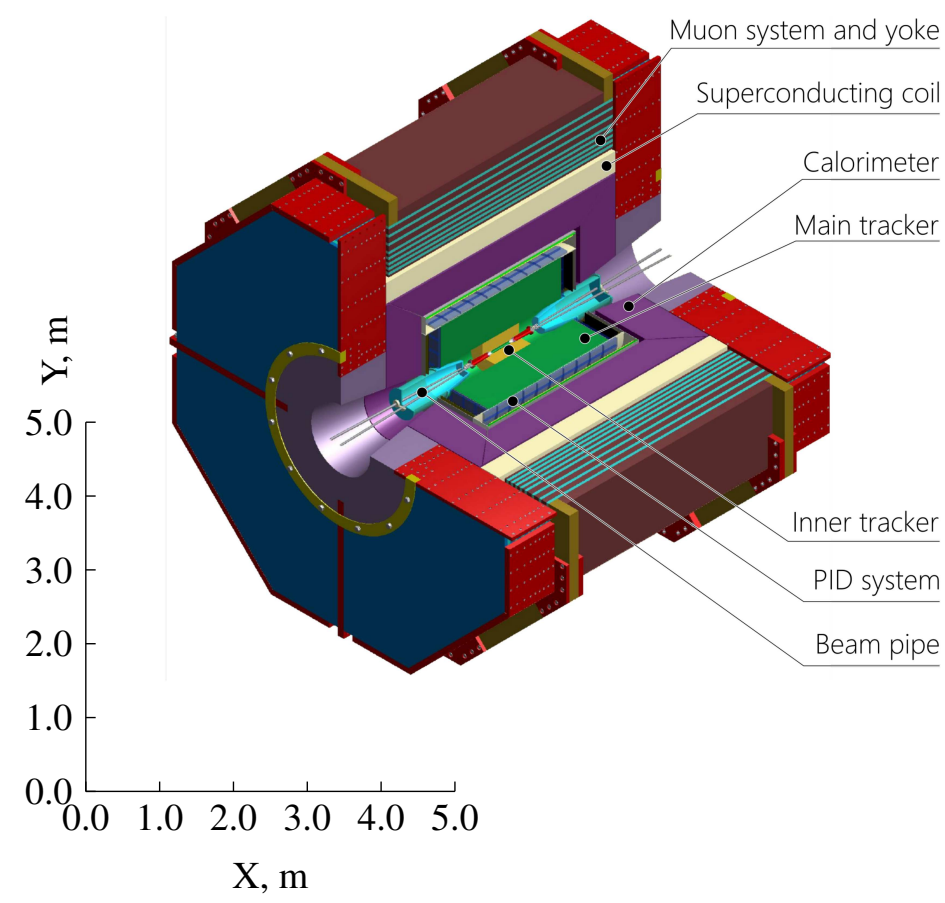

Figure 3: The detector conceptual scheme for SCT Factory in Novosibirsk.

on hexagonal drift cell with size $6 \div 7.5 \mathrm{~mm}, 41$ layers combined into 10 super-layers with alternating axial and stereo layers [13]. The second proposal is called "ultra low mass DC" with rectangular cell $7.2 \times 9.3 \mathrm{~mm}^{2}, 64$ stereo layers, total number of very thin wires is about 100000 [14]. In both cases $R \& D$ for new readout electronics is needed. If the readout electronics will provide the cluster counting it could help to improve the $\frac{d E}{d x}$-resolution approximately by two times and extend the momentum range for reliable particle identification with help of the trackig system. A dedicated PID system is needed to provide $\pi / K$ and $\mu / \pi$-separation with more than three standard deviations $(\sigma)$ in momentum ranges $0.6-2.5 \mathrm{GeV} / c$ and $0.2-1.2 \mathrm{GeV} / c$, respectively. The Focusing Aerogel RICH (FARICH) technique is able to meet these requirements as was demonstrated with simulation and the prototype beam test at CERN in $2012[15,16]$. Recent results on FARICH R\&D are presented in [17]. However alternative PID options such like DIRC, ToF (Time-of-Flight) and ToP (Time-of-Propagation) or aerogel Cherenkov threshold counters ASHIPH (Aerogel, Shifter, PHotomultiplayer) are also considered [18, 19]. The main tasks of electromagnetic calorimeter are to provide good $\pi^{0} / \gamma$-separation and $\gamma$ detection with $\mathrm{E}_{\gamma}=10 \div 3000 \mathrm{MeV}$. Also the calorimeter should be fast ( $\sigma_{t} \leqslant 1 \mathrm{~ns}$ and small shaping time) to suppress beam background and pileup noise. Therefore as a base option the calorimeter based on pure CsI crystals with decay time about $36 \mathrm{~ns}$ is considered. The recent R\&D at BINP shows that light output from the CsI-crystals could be increased almost by 6 times by using 4 avalanche photodiodes (APD) and wavelength shifter (WLS) with specific shapes [20]. To separate muons from hadrons the muon system placed in the 9 gaps of the yoke iron will be used. The option of the muon system for SCT factory project based on scintillating plastic and WLS fibre coupled to SiPMs (Silicon Photomultiplayer) is being developed in LPI (Moscow). Some details and R\&D progress are presented in [21]. 


\section{Summary}

The Super Charm-Tau Factory is one of the five megascience-class projects approved for implementation by the Russian government. The construction of the new electron-positron collider is a flagship project for the Budker Institute of Nuclear Physics (BINP). The physics program of the project will compliment the other current and future High Energy Physics experiments such as Belle II, LHCb and FCC [3]. Expected parameters of the collider and detector allow us to significantly increase the sensitivity of the experiment for new physics searches beyond the Standard Model. The process of forming an international collaboration to perform a broad physics program, develop final designs of the collider and the detector, and subsequently commence construction is underway. R\&D with prototyping for several systems of the collider and detector have been started in BINP and in several foreign institutes.

\section{References}

[1] Super Charm Tau Factory, Conceptual Design Report, vol. 1, BINP SB RAS, Novosibirsk 2018, https://ctd.inp.nsk.su/wiki/images/4/47/CDR2_ScTau_en_vol1.pdf

[2] Qin Q., Duan Z., Huang N. et al., Proceedings of IPAC' 10 (Kyoto, Japan,) Beam Dynamic Issues in the BEPCII Luminosity Comissioning, p. 1560

[3] Physics Briefing Book, Input for the European Strategy for Particle Physics Update 2020, CERN-ESU-00430 September 2019, http: / /cds. cern. ch/record/2691414

[4] A.V. Bobrov, A.E. Bondar, Nucl. Phys. B (Proc. Suppl.) 225-227 (2012) 195-197

[5] A.V. Bobrov, A.E. Bondar, Nucl. Phys. B (Proc. Suppl.) 253-255 (2014) 199-201

[6] D.Epifanov, "Measurement of Michel parameters in tau decays at the Super Charm-Tau factory with polarized electron beam", Joint Workshop on future Charm-Tau Factory 24-28 September 2019, RAS (Moscow)

[7] M. Zobov et al., Phys. Rev. Lett. 104, 174801 (2010)

[8] K. Akai, K. Furukawa, H. Koiso, Nucl. Instr. and Meth. A 907 (2018) 188-199

[9] I. Koop et al., "Longitudinal Polarization in Novosibirsk c-tau factory", Joint Workshop on future Charm-Tau Factory 24-28 September 2019, RAS (Moscow)

[10] P. Piminov, Phys. of Part. and Nucl. Lett. Vol. 15 No. 72018 732-736

[11] T.V. Maltsev et al., EPJ Web Conf., 212 (2019) 01011

[12] L. Shekhtman, F. Ignatov and V. Tayursky, EPJ Web Conf., 212 (2019) 01009

[13] K. Todyshev, "Drift chamber R\&D progress in Novosibirsk", Joint Workshop on future Charm-Tau Factory 24-28 September 2019, RAS (Moscow)

[14] F. Grancagnolo, "State-of-the-art of Drift chambers", CREMLIN WP7 "Super c-tau factory workshop" 26-27 of May 2018 BINP (Novosibirsk)

[15] A.Yu. Barnyakov et al., Nucl. Instr. and Meth. A 732 (2013) 352-356.

[16] A.Yu. Barnyakov et al., Nucl. Instr. Meth. A 766235 (2014)

[17] A.Yu. Barnyakov et al., Nucl. Instr. Meth. A (2019), article in press https://doi.org/10.1016/j.nima.2019.05.088

[18] A.Yu. Barnyakov et al., EPJ Web Conf., 212 (2019) 01012

[19] A.Yu. Barnyakov et al., "Particle identification system for the Super CharmâĂŞTau Factory at Novosibirsk”, Nucl. Inst. and Meth. A (2019) 162352, https://doi.org/10.1016/j.nima.2019.162352.

[20] Ekaterina Prokhorova, EPJ Web Conf., 212 (2019) 01007

[21] Timofey Uglov, EPJ Web Conf., 212 (2019) 01010 